\title{
Dynamic Deformation Electron-Phonon Interaction in Disordered Bulk Semiconductor
}

\author{
Pushpendra Tripathi ${ }^{* *}$, Syed Sikandar Zulqarnyn Ashraf ${ }^{1}$, Syed Tahir Hasan ${ }^{2}$, Ami Chand Sharma ${ }^{3}$ \\ ${ }^{1}$ Centre of Excellence in Materials Science (Nanomaterials), Department of Applied Physics, Zakir Husain College of Engineering \& \\ Technology, Aligarh Muslim University, Aligarh, India; ${ }^{2}$ Physics Department, Shibli National Post Graduate College, Azamgarh, India; \\ ${ }^{3}$ Physics Department, Faculty of Science, The Maharaja Sayajirao University of Baroda, Vadodara, India. \\ Email: "ptrip71@yahoo.com
}

Received September $28^{\text {th }}, 2011$; revised November $4^{\text {th }}, 2011$; accepted November $12^{\text {th }}, 2011$

\begin{abstract}
We present our theoretical investigations on the effects of disorder on the electron-phonon interaction in semiconducting GaAs system. Both the temperature ( $T$ ) and disorder (electron mean free path $l$ ) dependences of the electron-phonon scattering rate have been determined. On consideration of the dynamic screening, we find a significant change in the temperature exponent as well as the pre factor $\alpha$ from the earlier reported temperature power law dependence result $\alpha T^{6}$ obtained under static screening. Also the dynamic screening makes a noticeable change in the character of the dependence of scattering rate on the mean free path from the static screening result.
\end{abstract}

Keywords: Electron-Phonon Scattering Rate; Dynamic Screening; Deformation Potential

\section{Introduction}

Electron-phonon interaction $(e-p h)$ has drawn immense interest in the recent past [1-7]. The e-ph interaction in disordered metals has been extensively studied theoretically, by a good number of authors for over three decades, but quite different predictions were concluded [8]. For example, values for the temperature exponent $(p)$ ranging from 2 to 4 have been predicted. Experimentally, the temperature dependence of $1 / \tau_{p h}$ reported by various measurements on various material systems is not always in agreement with one another. This issue becomes even more delicate when one is concerned with the disorder behavior of $1 / \tau_{p h}$. On one hand, theories have predicted distinctly different disorder dependence of $1 / \tau_{p h}$, and on the other hand, few experiments in literature have succeeded in reporting a clear dependence of $1 / \tau_{p h}$ on disorder. The electron-phonon interaction in disordered systems is due to two process: a) the pure electron-phonon scattering (the only mechanism of the electron-phonon interaction in pure metals), and b) the inelastic electron scattering from vibrating impurities (defects, boundaries, etc). The last mechanism combined with the elastic electron scattering and electron-phonon scattering, generates a variety of interference processes [9].

Interaction between electrons and phonons is strongly affected by the disorder of the electron system and, there-

${ }^{*}$ Corresponding author. fore, the problem is commonly divided into two special cases: the pure and impure (or diffusive) limit. The crossover between these two limits is defined by $q l=1$, where $q$ is the phonon wave vector and $l$ is the electron mean free path. When the whole phonon system is consi- dered, then the phonon wave vector can be conveniently replaced by the thermal phonon wave vector, $q_{T}=$ $\left(K_{B} T\right) / \hbar u$ where $T$ is the temperature of the lattice and $u$ is the sound velocity. The $e-p h$ interaction in disordered semiconductors is much more complicated than in pure bulk systems. Despite the progress in theory the issue has not been settled completely yet [10]. Sergeev et al. have obtained analytical results on the $e-p h$ scattering rates via the Deformation Potential (DP) for single valley disordered semiconductor bulk and nanostructures [7]. They predict that the e-ph energy relaxation is strongly enhanced when the system enters from the pure limit $(q l>1)$ to the diffusive limit $(q l<1)$. Various temperature $(T)$ and mean free path $(l)$ dependences have been determined in the static screening condition. The behavior is the opposite in comparison to metals where it is well known, since the pioneering work by Pippard [11], that the disorder of the electron system tends to suppress the $e$-ph energy relaxation. In semiconductors, due to small electron density, the $e-p h$ interaction can be described by deformation potential coupling constants, which do not depend on the electronic variables, while in metals the coupling strongly depends on the electron momentum [12]. 
Chow et al. studied that the phonon emission power in dirty sample is significantly enhanced due to disorder induced change in the screening from static to dynamic [13]. Other studies have also highlighted the significance of dynamic screening [14-17]. Yu et al. [18] presented a detailed experimental investigation of the effects of disorder on the electron-phonon interaction in semiconducting InN system. Both the temperature $(T)$ and disorder (electron mean free path $l$ ) dependences of the electron-phonon scattering time $\tau_{e-p h}$ have been determined from the weak localization analysis. They have found $\tau_{e-p h}^{-1}$ exhibits a tendency to change gradually from that the characteristic dependence $\tau_{e-p h}^{-1} \propto T^{3} l^{0}$ in the pure limit to the form of $T^{2} l^{-1}$ as the films become disordered. Their observation gives clear evidence for the substantial enhancement of the electron-phonon coupling in semiconductor structures due to elastic electron scattering. Recently, J. T. Muhonen et al. have focused on the signifycance of the electron-phonon energy loss rate in a manyvalley semiconductor system due to lattice mismatch induced strain [19].

In view of these aforementioned points, we investigated the electron phonon rate in disordered three dimensional electron gas (3DEG) confined to GaAs system interacting with bulk (3D) phonons in the full diffusive limit incorporating the dynamic DP, to determine by what degree and character the dynamical behavior of these functions influences the electron-phonon scattering rate. The paper is organized as follows: Section 2 describes the formalism; Section 3 contains the results and discussion and lastly; Section 4 concludes the work.

\section{Formalism}

The e-ph relaxation rate, $\tau_{e-p h}^{-1}$ is determined by the following kinetic equation [17]:

$$
\frac{1}{\tau_{e-p h}}=-\frac{\delta}{\delta n(\varepsilon)} \frac{\mathrm{d} n(\varepsilon)}{\mathrm{d} t}
$$

where $n(\varepsilon)$ is the non equilibrium electron distribution function in a spatially uniform system. In equilibrium, phonon and electron distribution functions respectively are $N_{\omega}=N_{\omega_{q}}^{e q}$ and $n_{\varepsilon}=n_{\varepsilon_{q}}^{e q}$, and the $\tau_{e-p h}^{-1}$ at the Fermi surface $(\varepsilon=0)$ is given by

$$
\frac{1}{\tau_{e-p h}}=\frac{4 \tau}{\hbar} \int \frac{\mathrm{d} \mathbf{q}}{(2 \pi)^{3}} \frac{\gamma^{2}}{\left|\varepsilon\left(q, \omega_{q}\right)\right|^{2}} \Re\left\{\frac{\xi\left(q, \omega_{q}\right)}{1-\xi\left(q, \omega_{q}\right)}\right\}\left\{N_{\omega_{q}}^{e q}+n_{\varepsilon}^{e q}\right\}
$$

where $\tau$ is the relaxation time for electron-impurity scattering, $\hbar$ is the Planck's constant, $\boldsymbol{q}$ is the $3 \mathrm{D}$ phonon momentum, $v=m k_{F} / \pi^{2} \hbar^{2}$ is the $3 \mathrm{D}$ electronic density of states; $m *$ is the effective electronic mass,
$\gamma=D_{0}(q \cdot e) /\left(2 r w_{q}\right)^{1 / 2}$ is the vertex of the unscreened electron acoustic phonon DP interaction, $D_{0}$ is the coupling constant of DP, $\boldsymbol{e}$ is the polarization vector, $\rho$ is the density of the material, $\omega_{q}=|\boldsymbol{q}| u$, is the linear phonon dispersion, $u$ is the longitudinal sound velocity,

$N_{\omega_{q}}^{e q}=\left[\exp \left(\hbar \omega_{q} / k_{B} T\right)-1\right]^{-1}$ is the phonon distribution function at equilibrium, $n_{\varepsilon}^{e q}=\left[\exp \left(\hbar \omega_{q} / k_{B} T\right)+1\right]^{-1}$ is the electron distribution function at equilibrium, $\varepsilon\left(\omega_{q}, q\right)$ is the dynamic dielectric function, and

$\Re\left[\xi\left(\omega_{q}, q\right) /\left(1-\xi\left(\omega_{q}, q\right)\right)\right]$ is the real part of the polarization function that has been obtained by simplifying the response functions [7]. The dynamic screening of DP is described in terms of the dynamic dielectric function which is given by;

$$
\varepsilon\left(q, \omega_{q}\right)=1+V^{0}(q) \Pi\left(q, \omega_{q}\right)
$$

in which $V^{0}(q)=4 \pi e^{2} / \varepsilon_{0} q^{2}$ is the 3D Fourier transform of the bare Coulomb potential, $e$ is the electronic charge, $\varepsilon_{0}$ is the background dielectric constant, and $\Pi\left(q, \omega_{q}\right)$ is the polarization operator. In the limit of small $q$ and $\omega$, the dielectric function in the diffusive limit is given by [20].

$$
\varepsilon\left(q, \omega_{q}\right)=1+\frac{4 \pi e^{2}}{\varepsilon_{0} q^{2}} \frac{v D q^{2}}{-i \omega_{q}+D q^{2}}
$$

where $D=v_{F}^{2} \tau / 3$ is the $3 \mathrm{D}$ diffusion constant. The dynamic polarization function for $3 \mathrm{DEG}$ is obtained as [7];

$$
\xi\left(q, \omega_{q}\right)=\frac{1}{q l} \tan ^{-1} \frac{q l}{\left(1-i \omega_{q} \tau\right)}
$$

For, $\omega_{q} \quad \square \quad D q_{l l}^{2}$, the $\omega_{q}$-dependence of the dielectric function in Equation (4) can be dropped to yield the statically screened dielectric function,

$$
\varepsilon(q)=1+\frac{k^{2}}{q^{2}}
$$

where $k^{2}=4 \pi e^{2} v$. The dynamic polarization function in Equation (5) in the static limit reduces to

$$
\xi(q)=\frac{\tan ^{-1} q l}{q l}
$$

The electron-phonon rates have been calculated in Ref. [7] from Equation (2) by substituting, static dielectric function $\varepsilon\left(q_{l l}\right)$, which is a result of static polarization function $\xi\left(q_{l l}\right)$. In terms of dimensionless parameters; $x=\hbar q u / k_{B} T, y=k_{B} T l / \hbar u$ and $z=k_{B} T / \hbar u k_{2}$, where $k_{B}$ and $\hbar$ are the Boltzmann's and Planck's constants respectively, $k$ is the inverse screening length [17], the Equation (2) takes the form; 


$$
\begin{aligned}
\frac{1}{\tau_{e-p h}}= & \frac{D_{0}^{2} k_{B}^{3} T^{3}}{\pi^{2} u^{3} \hbar^{4} v_{F} \rho} \\
& \cdot \int_{0}^{\infty} x^{2} \mathrm{~d} x\left(\frac{x^{2} z^{2}\left(x^{4} y^{4}+9 x^{2} y^{2}\left(u / v_{F}\right)^{2}\right)}{x^{2} z^{2}\left\{x^{4} y^{4}+9 x^{2} y^{2}\left(u / v_{F}\right)^{2}\right\}+x^{4} y^{4}}\right)^{2} \\
& \cdot \operatorname{Re}\left\{\frac{x y \tan ^{-1}\{x y\}}{x y-\tan ^{-1}\{x y\}}\right\}\left\{N_{x}^{e q}+n_{x}^{e q}\right\}
\end{aligned}
$$

The analytical result under strong screening for the case $T<\hbar u / K_{B} l$ is reproduced here from Ref. 7, for the ease of reference,

$$
\frac{1}{\tau_{e-p h}}=\frac{3 \pi^{6}}{4} \frac{D_{0}{ }^{2} v K_{B}{ }^{6} T^{6}}{\rho u^{2} k^{4} p_{F}^{2} l u^{5} \hbar^{4}}
$$

\section{Results and Discussion}

The approximate analytical evaluation of Equation (1) cannot be a replacement for a full numerical treatment of the concerned equation. In order to see the complete effects of dynamic DP on temperature and disorder we took recourse to numerical computation, as in Ref. 7 only approximate analytical results in the static weak and strong screening and impurity limit respectively have been obtained. The electron-phonon scattering rates is computed as a function of $T, l$ and carrier density $(\mathrm{N})$ for disordered 3DEG confined to GaAs system that has been modeled in terms of the following material parameters; $D_{0}=8.0 \mathrm{eV}$, $\varepsilon_{0}=12.5, u=5.2 \times 10^{5} \mathrm{~cm} / \mathrm{S}, \rho=5.2 \mathrm{gm} / \mathrm{cm}^{3}, \mathrm{~m}^{*}=$ $0.067 \mathrm{~m}$, and electron density $N=5 \times 10^{17} \mathrm{~cm}^{-3}$. This electron density corresponds to the Bloch-Gruneisen temperature limit of $19.50 \mathrm{~K}$. Figure 1 presents (a) the extracted exponent of temperature $p$ and (b) the e-ph scattering rate $\left(\tau_{e-p h}^{-1}\right)$ for a fixed value of mean free path $(l=10 \mathrm{~nm})$ as a function of temperature. The curve A displays the analytical result of Equation (9) under static screening case. Curves B and C of Figure 1(b), has been obtained by computing Equation (2) and Equation (8) incorporating the static and dynamic dielectric functions respectively. We observe that the power exponent (p) of temperature varies from $\mathrm{T}^{6.134}$ to $\mathrm{T}^{3.95}$, for the lower and upper values of plotted range of temperature. It is observed from the Figure 1(b) that the analytical curve A and the numerical dynamic result $\mathrm{B}$ and static result $\mathrm{C}$ merge with each other for values of temperature less than $5 \mathrm{~K}$ but above this temperature the curves $\mathrm{B} \& \mathrm{C}$ together trace a different path from that of the analytical curve A. To investigate the behaviors of $e-p h$ rate with disorder, we plotted in Figure 2, the variation of electron-phonon scattering rate with mean free path $(l)$ for a fixed value of temperature $\mathrm{T}=10 \mathrm{~K}$. Curve A of Figure 2 is plotted for the analytical static case from Equation (9) and curves B and $\mathrm{C}$ are plotted for numerically static and dynamic case.

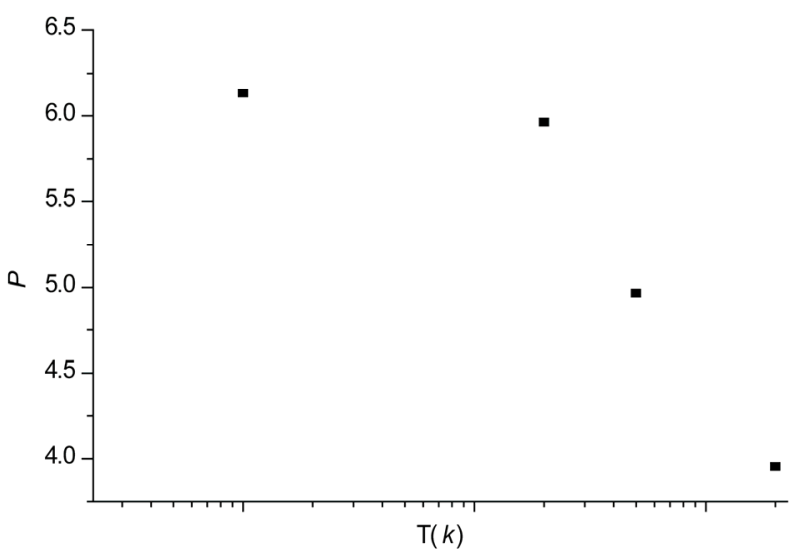

(a)

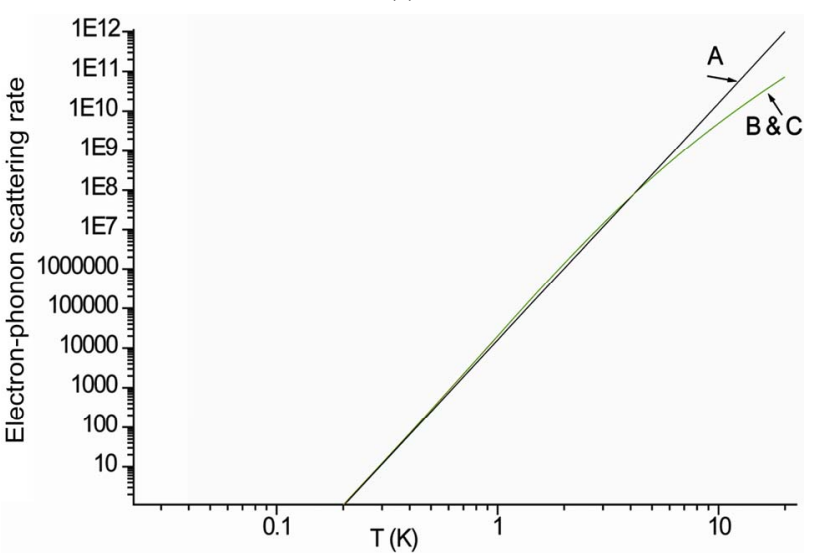

(b)

Figure 1. (a) Values of $p$ as a function of temperature (T); (b) Electron-phonon scattering rate $\left(\tau_{e-p h}^{-1}\right)$ versus temperature for electron mean free path $(I=10 \mathrm{~nm})$. Curve A displays the analytic result reported in [7]. Curves B \& C are plotted numerically by taking static and dynamic screening from Equations (2) \& (8), respectively.

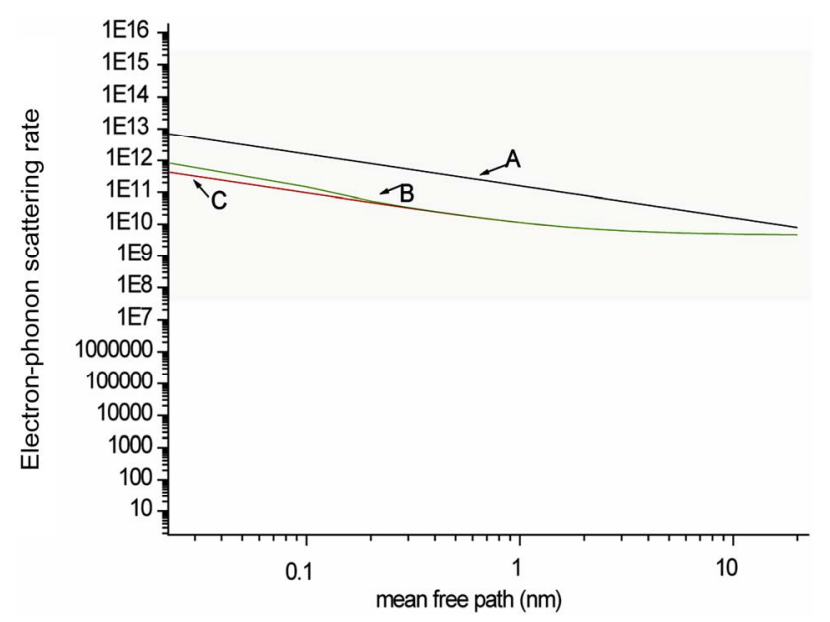

Figure 2. Electron-phonon scattering rate $\left(\tau_{e-p h}{ }^{-1}\right)$ as a function of mean free path $(I)$. Curve $A$ displays the analytic result reported in [7] at $T=10 \mathrm{~K}$. Curves $B$ \& $C$ are plotted numerically by taking static and dynamic screening from Equations (2) \& (8), respectively. 
It is noticed from Figure 2 that the analytical curve A varies inversely with mean free path $(l)$ but the numerical curves $\mathrm{B} \& \mathrm{C}$ deviate from this strict inverse behavior. These curves A, B \& C come close together for higher values of $(l)$ and separate out from each other in the opposite limit of decreasing $(l)$. The variation of the electron-phonon scattering rate with carrier density $\mathrm{N}$ can be seen in the Figure 3 which is plotted at a temperature of $10 \mathrm{~K}$. Again the analytical curve A is observed to vary inversely with carrier density $(\mathrm{N})$. However, the numerical curves B \& C departs from this linear behavior as has been the case in the above two figures. The differences between the analytical and numerical static and numerical dynamic is best highlighted in this figure. All this three figures corroborate the results produced in each of them. Therefore, clearly this comparative theoretical study of the electron-phonon scattering rate with the static and dynamic screening shows that the dynamic screening cannot be ignored as it significantly modifies the magnitude and behavior of electron-phonon scattering rate in disordered bulk semiconductor system.

\section{Conclusion}

The temperature and mean free path variation of $\left(\tau_{e-p h}{ }^{-1}\right)$ depend strongly on disorder. The character of the electronphonon interaction via the deformation potential (DP) is affected significantly by the inclusion of the dynamic screened DP and the polarization operator. On inclusion of dynamic screened DP the temperature exponent is enhanced at lower temperatures and reduces at higher temperatures from that of the analytical $\mathrm{T}^{6}$ result obtained under the statically screened DP approximation. The magnitude is also reduced at higher temperature. Further the dynamically screened electron-phonon scattering rate is

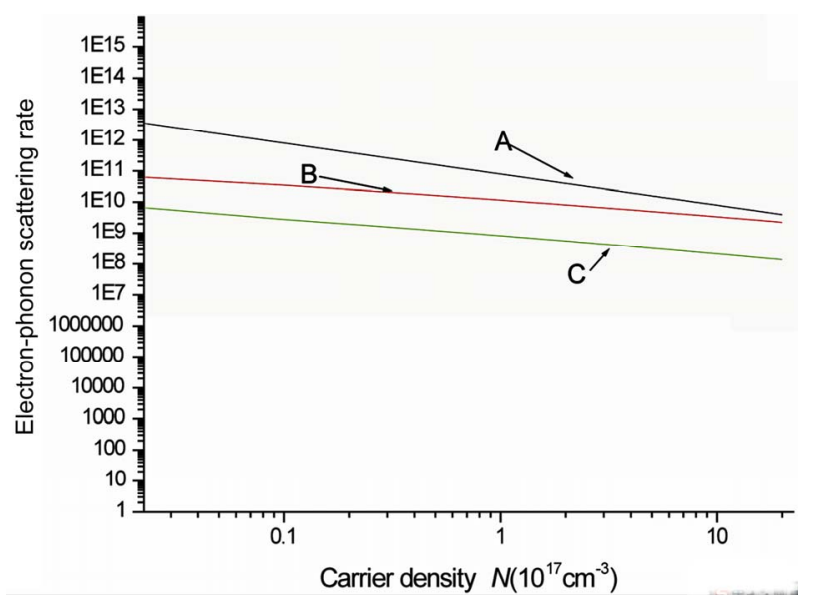

Figure 3. Electron-phonon scattering rate $\left(\tau_{e-p h}{ }^{-1}\right)$ as a function of electron density ( $N)$. Curve A displays the analytical result reported in [7] at $T=10 \mathrm{~K}$ and $l=10 \mathrm{~nm}$. Curves $B$ and $C$ are plotted numerically by taking static and dynamic screening from Equations (2) \& (8), respectively. found to decrease with mean free path too, in comparison to the earlier reported analytical calculation under static screening case. We therefore conclude from this complete numerical computation of the electron-phonon scattering rate that dynamic nature of screened DP cannot be ignored as it significantly affects the calculations and therefore governs the temperature and mean free path dependence of the electron-phonon scattering rate.

\section{Acknowledgements}

The authors acknowledge with thanks the financial support through the research projects sanctioned by DST, New Delhi and DAE BRNS, Mumbai.

\section{REFERENCES}

[1] A. Sergeev and V. Mitin, "Effect of Electronic Disorder on Phonon-Drag Thermopower," Physical Review B, Vol. 61, No. 9, 2000, pp. 6041-6047. doi:10.1103/PhysRevB.61.6041

[2] S. Agan, O. A. Mironov, E. H. C. Parker, T. E. Whall, C. P. Parry, V. Y. Kashirin, Y. F. Komnik, V. B. Krasovitsky, B. Verkin and C. J. Emeleus, "Low-Temperature Electron Transport in $\mathrm{Si}$ with an MBE-Grown $\mathrm{Sb} \delta$ Layer," Physical Review B, Vol. 63, 2001, pp. 5402-5472. doi:10.1103/PhysRevB.63.075402

[3] M. E. Gershenson, D. Gong, T. Sato, B. S. Karasik and A. V. Sergeev, "Millisecond Electron-Phonon Relaxation in Ultrathin Disordered Metal Films at Millikelvin Temperatures," Applied Physics Letters, Vol. 79, No. 13, 2001, pp. 2049-2051. doi:10.1063/1.1407302

[4] O. Prus, M. Reznikov, U. Sivan and V. Pudalov, "Cooling of Electrons in a Silicon Inversion Layer," Physical Review Letters, Vol. 88, No. 1, 2002, pp. 6801-6804. doi:10.1103/PhysRevLett.88.016801

[5] P. Kivinen, A. Savin, M. Zgirski, P. Torma, J. Pekola, M. Prunnila and J. Ahopelto, "Electron-Phonon Heat Transport and Electronic Thermal Conductivity in Heavily Doped Silicon-On-Insulator Film," Journal of Applied Physics, Vol. 94, No. 5, 2003, pp. 3201-3025. doi:10.1063/1.1592627

[6] A. K. Meikap, Y. Y. Chen and J. J. Lin, "Anomalous Temperature and Disorder Dependences of ElectronPhonon Scattering Rate in Impure $\mathrm{V}_{1-x} \mathrm{Al}_{x}$ Alloys," Physical Review B, Vol. 69, No. 21, 2004, pp. 2202-2205. doi:10.1103/PhysRevB.69.212202

[7] A. Sergeev, M. Y. Reizer and V. Mitin, "Deformation Electron-Phonon Coupling in Disordered Semiconductors and Nanostructures," Physical Review Letters, Vol. 94, No. 13, 2005 pp. 6602-6605. doi:10.1103/PhysRevLett.94.136602

[8] C. Y. Wu, W. B. Jian and J. J. Lin, "Electron-Phonon Scattering Times in Crystalline Disordered Titanium Alloys between 3 and 15 K," Physical Review B, Vol. 57, No. 18, 1998, pp. 11232-11241. doi:10.1103/PhysRevB.57.11232

[9] N. G. Ptitsina, G. M. Chulkova, K. S. Il'in, A. V. Sergeev, 
F. S. Pochinkov and E. M. Gershenzon, "Electron-Phonon Interaction in Disordered Metal Films: The Resistivity and Electron Dephasing Rate," Physical Review B, Vol. 56, No. 16, 1997, pp. 10089-10096.

[10] J. J. Lin and J. P. Bird, "Recent Experimental Studies of Electron Dephasing in Metal and Semiconductor Mesoscopic Structures," Journal of Physics: Condensed Matter, Vol. 14, No. 18, 2002, pp. R501-R596. doi:10.1088/0953-8984/14/18/201

[11] A. B. Pippard, "Ultrasonic Attenuation in Metals," Philosophical Magazine, Vol. 46, 1955, pp. 1104-1114.

[12] M. Prunnila, P. Kivinen, A. Savin, P. Torma and J. Ahopelto, "Intervalley Scattering-Induced Electron-Phonon Energy Relaxation in Many-Valley Semiconductors at Low Temperatures," Physical Review Letters, Vol. 95, No. 20, 2005, pp. 206602-206605. doi:10.1103/PhysRevLett.95.206602

[13] E. Chow, H. P. Wei, S. M. Grivin, W. Jan and J. E. Cunningham, "Effect of Disorder on Phonon Emissions from a Two-Dimensional Electron Gas in $\mathrm{GaAs} / \mathrm{Al}_{x} \mathrm{Ga}_{1-x} \mathrm{As}$ Heterostructures," Physical Review B, Vol. 56, No. 4, 1997, pp. R1676-R1679. doi:10.1103/PhysRevB.56.R1676

[14] P. Tripathi and B. K. Ridley, "Dynamics of Hot-Electron Scattering in GaN Heterostructures," Physical Review B, Vol. 66, No. 19, 2002, pp. 195301-195310. doi:10.1103/PhysRevB.66.195301
[15] S. C. Lee and I. Galbraith, "Intersubband and Intrasubband Electronic Scattering Rates in Semiconductor Quantum Wells," Physical Review B, Vol. 59, No. 24, 1999, pp. 15796-15805. doi:10.1103/PhysRevB.59.15796

[16] E. Chow, H. P. Wei, S. M. Grivin and M. Shayegan, "Phonon Emission from a 2D Electron Gas: Evidence of Transition to the Hydrodynamic Regime," Physical Review Letters, Vol. 77, No. 6, 1996, pp. 1143-1146. doi:10.1103/PhysRevLett.77.1143

[17] S. S. Z. Ashraf, P. Tripathi, A. C. Sharma and S. T. Hasan, "Electron-Phonon Relaxation in Disordered Two-Dimensional Electron Gas with Dynamically Screened Deformation Potential," Journal of Physics: Condensed Matter, Vol. 21, No. 2, 2009, p. 025504. doi:10.1088/0953-8984/21/2/025504

[18] X. Z. Yu, Y. Yang and W. Pan, "Electron-Phonon Interaction in Disordered Semiconductors," Applied Physics Letters, Vol. 92, No. 9, 2008, pp. 092106-092108.

[19] J. T. Muhonen, et al. "Strain Dependence of ElectronPhonon Energy Loss Rate in Many-Valley Semiconductors," Applied Physics Letters, Vol. 98, No. 18, 2011, pp. 182103-182105. doi:10.1063/1.3579524

[20] G. D. Mahan, "Many Particle Physics," 2nd Edition, Plenum, New York, 1990. doi:10.1007/978-1-4613-1469-1 